\title{
Developing a Prediction Score for the Diagnosis of Malignant Pleural Effusion: MPE Score
}

\section{Chaichana Chantharakhit*, Nantapa Sujaritvanichpong}

\begin{abstract}
Background: The objective of this study was to develop a diagnostic prediction model for diagnosis of malignant pleural effusion (MPE) from pleural fluid cytology (MPE score). Materials and Methods: Retrospective analysis of pleural fluid cytology was conducted in patients with MPE between 2018 and 2020. Multivariable logistic regression was used to explore the potential predictors. The selected logistic coefficients were transformed into a diagnostic predictive scoring system. Internal validation was done using the bootstrapping procedure. Results: The data of pleural fluid cytology from 155 MPE patients were analyzed. Seventy-eight positive pleural cytology patients were found $(50.32 \%)$. Lung cancer was the cancer most commonly sent for pleural fluid testing, with $66.67 \%$ positive cytology. The predictive indicators included pleural fluid protein $>4.64 \mathrm{~g} / \mathrm{dL}$, pleural fluid LDH $>555 \mathrm{IU} / \mathrm{L}$, and pleural fluid sugar $>60 \mathrm{mg} / \mathrm{dL}$. Lung mass from imaging and double tap for pleural cytology were used for the derivation of the diagnostic prediction model. The score-based model showed that the area under the receiver operating characteristic curve was 0.74 (95\% CI 0.66-0.82). The developed MPE score ranged from zero to 17 . The cut-off point was 15 with $88.31 \%$ of specificity, $37.18 \%$ of sensitivity, positive predictive value of 0.76 , and negative predictive value of 0.58 . The measurement of the calibration was illustrated using a calibration plot ( $\mathrm{p}$-value $=0.49$ for the Hosmer-Lemeshow based goodness of fit). Internal validation with 1,000 bootstrap resampling showed a good discrimination. Conclusions: The MPE score, as the diagnostic prediction model can be used in planning for more efficient diagnosis of MPE in patients with cancer under MPE.
\end{abstract}

Keywords: MPE score- pleural fluid cytology- diagnostic prediction model- malignant pleural effusion

Asian Pac J Cancer Prev, 23 (1), 25-31

\section{Introduction}

Malignant pleural effusion (MPE) refers to finding cytology pleural fluid caused by the metastasis of malignant mesothelioma, which is mostly due to lung cancer in men and breast cancer in women (Psallidas et al., 2016; Agrawal et al., 2015; Mongardon et al., 2011; Aydin et al., 2009). The MPE is also the cause of exudative pleural effusion from $42 \%$ up to $77 \%$ (Valdes et al., 1996).

Diagnosis of MPE through cytology initially showed $60 \%$ of positive cytology depending on the type of cancer cells and cancer severity (Antonangelo et al., 2015; Loddenkemper and Boutin, 1993). Later, the diagnostic accuracy of MPE was improved by using pleuroscopy to enhance the efficiency of testing metastasis to the pleura (Ali et al., 2019; Ferreiro et al., 2017). However, this method is an invasive procedure. Therefore, less invasive ones are used such as metabolic imaging with 18-fluorodeoxy glucose positron emission tomography (FDG-PET). The sensitivity was increased to $90 \%$ (Nakajima et al.,2015; Toaff et al.,2005); nonetheless, this method could not determine the types of cancer cells.
In addition, epigenetic analysis of the pleural fluid was used to distinguish malignant DNA from methylation-specific PCR (MSP). This could help the diagnosis of MPE and efficiently specify the types of cancer cells (Herman et al., 1996; Brock et al., 2005; Zhang et al., 2007). Nevertheless, this method is expensive and is not used widely.

Cytology is still a key method with $60 \%$ sensitivity depending on the type of cancer (Johnston, 1985; Starr and Sherman, 1991; and Hsu, 1987). Mostly, positive cytology pleural fluid is found in lung cancer and breast cancer. According to studies (Garcia et al., 1994; Desai and Lee, 2017), the repetition pleural fluid cytology can increase the diagnostic opportunities by $24 \%$. However, more than double of the repetition is impractical for the diagnosis of MPE (Garcia et al., 1994). Thus, pleuroscopy is also required for confirmation to conduct a pleural biopsy, which is an invasive procedure.

The clinical features and pleural fluid profile should be used to assist MPE diagnosis as a routine clinical practice and a diagnostic prediction score to facilitate decision-making on whether to wait for cytology results 
or perform an invasive procedure for efficient and rapid MPE diagnosis. This is because some hospitals still have limited diagnosing capabilities, or patients may have to wait for the cytologic results for weeks. Moreover, not all hospitals have the facilities to perform pleuroscopy, which make the test inaccessible for many patients. Therefore, this diagnostic research aimed to develop a diagnostic prediction model to help the decision-making in the diagnosis of MPE and plan appropriate and efficient diagnostic guidelines in the future.

\section{Materials and Methods}

Clinical characteristics of patients with suspected MPE and cytology results of pleural fluid in Buddhasothorn Hospital, Chachoengsao, and Thailand were collected between 2018 and 2020. The inclusion criteria were as follows:

1) Patients older than 18 years.

2) The results of the pleural fluid comprised of biochemical tests and key serum tests, i.e., lactate dehydrogenase (LDH) and protein.

3) Cancer data based on the radiology findings.

The exclusion criteria consisted of:

1) No results of a pathological diagnosis in the case of negative cytology pleural fluid based on the cytologic results.

\section{Data analysis}

Step 1: The data were analyzed to find the potential factors in the diagnosis of positive cytology pleural fluid (MPE) using univariate regression analysis and multivariate logistic regression analysis.

Step 2: Multiple imputation for missing data: Three predictor variables (pleural fluid white blood cell, pleural fluid lymphocyte, pleural fluid sugar) had more than $10 \%$ missing values, which could lead to biased estimates of the diagnostic model with the complete-case analysis. Multiple imputation with chained equation via mi impute chained command was used to generate missing values prior to model derivation. The logit model was chosen for the imputation of multivariable missing predictors.

Step 3: The predictive variables from the multivariate logistic regression analysis were brought into the transformation of the risk score. A logistic regression coefficient was used to develop the MPE score to help diagnosing MPE.

Step 4: The area under the receiver operating characteristic curve (AUC) based on the MPE score for the diagnosis of MPE was calculated and showed the sensitivity, specificity, positive predictive value (PPV), negative predictive value (NPV), likelihood ratio for a positive test $(\mathrm{LR}+)$ and likelihood ratio for a negative test (LR -).

Step 5: The accuracy was tested through calibration curve and the Hosmer-Lemeshow goodness of fit test. Internal validation was tested by using the bootstrapping procedure (1,000 replicates).

This study was approved by the Institutional Review Board of Buddhasothorn Hospital under the codes BSHIRB 036/2563

\section{Results}

The data of the cytologic results of pleural fluid of 166 patients were collected. Eleven patients were excluded; including six patients with incomplete data of the biochemical tests and five patients did not have the pathological results to confirm the malignancy diagnosis. Therefore, the data were collected for 155 patients.

Seventy-eight patients $(50.32 \%)$ had positive cytology whereas seventy-seven patients $(49.68 \%)$ had negative cytology. In terms of the pathological diagnosis and among different cancers, lung cancer was the most frequent cancer $(61.9 \%)$ that needed the pleural fluid test. It was also the cancer with $66.67 \%$ of positive cytology (Table 1 ).

Based on the univariate analysis of the clinical characteristics and pleural fluid profile on MPE, it was found that lung mass detected by clinical imaging, lung cancer, breast cancer, and lung cancer with extrathoracic metastasis were the factors significantly affecting the predictive variables on MPE (Table2).

\section{Model development}

After analyzing the variable factors by univariate logistic regression analysis, the potential predictors affecting the diagnosis of MPE were selected for the multivariate logistic regression analysis of the scoring system derivation. The area under the receiver operating characteristic curve (AUC) for the final model was equal to 0.74 (95\% CI $0.66-0.82)$.

\section{Score transformation}

Each potential predictor in the multivariable model was assigned with a specific score derived from the logistic regression coefficient (Table 3 ). The scoring scheme had a total score ranging from zero to 17 . For the discriminative ability, the area under the parametric ROC curve for the score-based logistic regression model was equal to 0.74 (95\% CI 0.66-0.82) (Figure 1). The measurement of the calibration is illustrated with a calibration plot, and the p-value via the Hosmer-Lemeshow goodness of fit test is equal to 0.49 (Figure 2).

According to the sensitivity and specificity in each cut-off point, the point at 15 had $88.31 \%$ of specificity and $37.18 \%$ of sensitivity. This point displayed appropriate specificity that could be used as a diagnosis tool (Table

Table 1. Types of Cancer Confirming the Pathological Diagnosis with the Cytologic Results of the Pleural Fluid

\begin{tabular}{lccc}
\hline Types of Cancer & $\begin{array}{c}\text { Positive } \\
\text { Cytology (\%) }\end{array}$ & $\begin{array}{c}\text { Negative } \\
\text { Cytology (\%) }\end{array}$ & Total \\
\hline Lung & $64(66.67)$ & $32(33.33)$ & 96 \\
Breast & $10(38.46)$ & $16(61.54)$ & 26 \\
Colorectal & $0(0.00)$ & $4(100.00)$ & 4 \\
Lymphoma & $0(0.00)$ & $9(100.00)$ & 9 \\
Gastric & $1(14.29)$ & $6(85.71)$ & 7 \\
Prostate & $0(0.00)$ & $3(100.00)$ & 3 \\
Ovary & $1(20.00)$ & $4(80.00)$ & 5 \\
Head and Neck & $1(100.00)$ & $0(0.00)$ & 1 \\
Melanoma & $1(25.00)$ & $3(75.00)$ & 4 \\
\hline
\end{tabular}



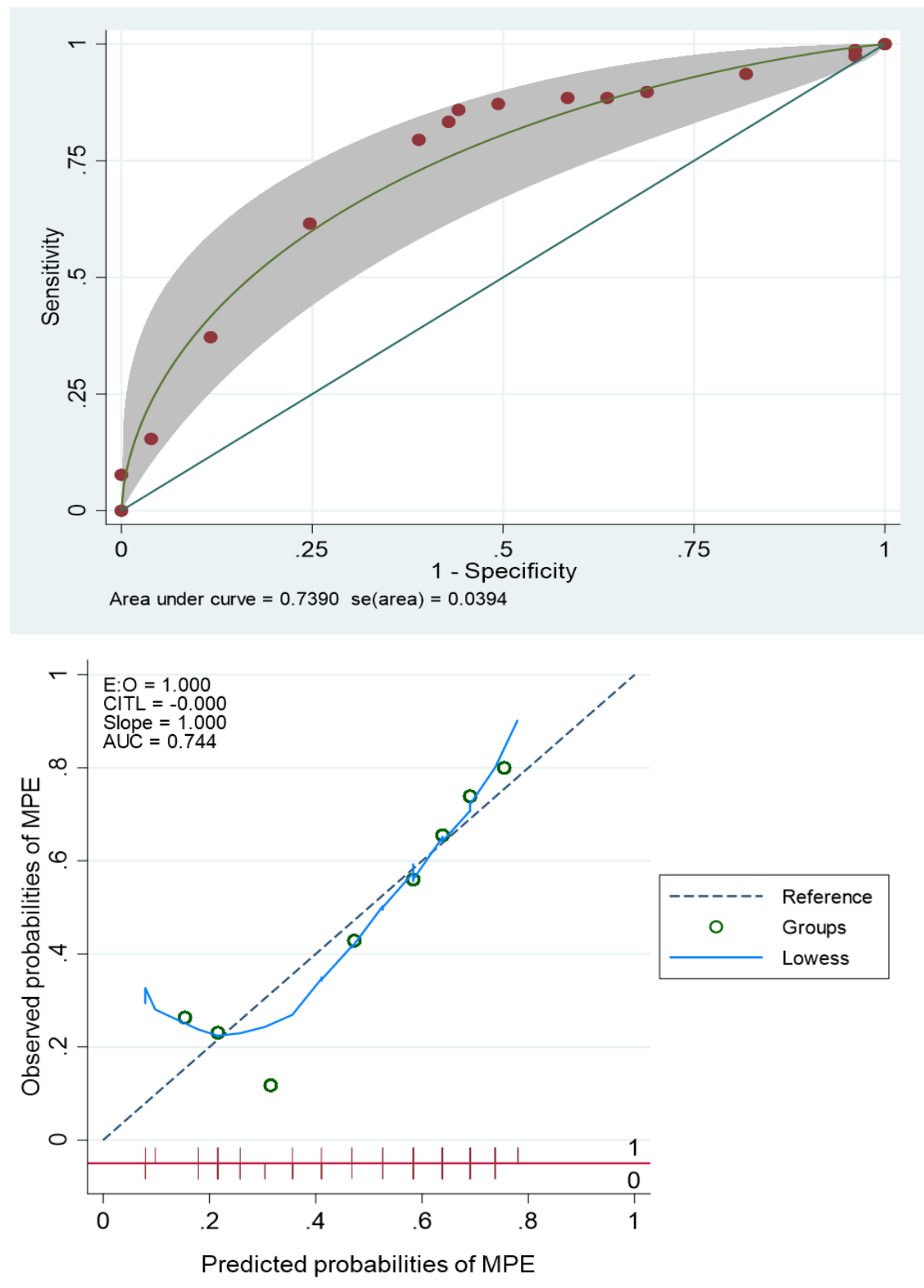

Figure 1. Performance of the Clinical Risk Score, Area under the Receiver Operating Characteristics Curve (AUC), and 95\% Confidence Band (Above). The calibration plots (pmcalplot) comparing the observed probabilities (y) and predicted probabilities (x) of the use of the MPE score to predict MPE (Below).

4). With the cut-off point of the MPE score at 15, to help the diagnosis of MPE, the odds ratio was equal to 3.18 (95\% CI 1.35-8.11; p-value 0.004), positive predictive value (PPV) was equal to 0.76 , negative predictive value (NPV) was equal to 0.58 , positive likelihood ratio ( $\mathrm{LR}+$ ) was equal to 3.18 , and negative likelihood ratio (LR-) was equal to 0.71 .

\section{Internal validation}

Through conducting the internal validation using the predictive model with 1,000 resampling bootstrap method data set, the mean of the AUC of the apparent curve was obtained equal to 0.75 , the test curve was equal to 0.72 (bootstrap estimator), and average estimates of the optimism curve was equal to 0.03 (Table 5).

\section{Discussion}

Pleural fluid found in malignant disease was due to two major conditions, i.e., paramalignant pleural effusion (PMPE) and malignant pleural effusion (MPE) (Wong et al., 1963; Epelbaum and Rahman, 2019). The PMPE is not a consequence of a malignant disease spreading to the pleura. The probability that an effusion is paramalignant is higher when the effusion is transudative, while MPE is exudative. Therefore, understanding the differentiation between PMPE and MPE is necessary.

There are studies on the use of the cancer ratio using the ratio of serum lactate dehydrogenase $(\mathrm{LDH})$ to adenosine deaminase (ADA) in pleural fluid. The ratios used were based on the cut-off level $>20$ to help diagnose the causes of exudative pleural fluid between benign and MPE. It was found that sensitivity and specificity were high because the relationship of the levels of serum LDH 
Table 2. Univariate Logistic Regression Analysis of MPE and Variable Factors

\begin{tabular}{|c|c|c|c|}
\hline Variables & Odds Ratio & 95\% Confidence Interval & p-value \\
\hline Pleural fluid protein $<4.64 \mathrm{~g} / \mathrm{dL}$ & 1 & reference & \\
\hline Pleural fluid protein $>4.64 \mathrm{~g} / \mathrm{dL}$ & 1.56 & $0.82-2.94$ & 0.171 \\
\hline Pleural fluid LDH $<555$ IU/L & 1 & reference & \\
\hline Pleural fluid LDH $>555 \mathrm{IU} / \mathrm{L}$ & 1.14 & $0.61-2.14$ & 0.686 \\
\hline Pleural fluid sugar $<60 \mathrm{mg} / \mathrm{dL}$ & 1 & reference & \\
\hline Pleural fluid sugar $>60 \mathrm{mg} / \mathrm{dL}$ & 2.01 & $0.83-4.88$ & 0.122 \\
\hline Low protein ratio $(<0.5)$ & 1 & reference & \\
\hline High protein ratio $(>0.5)$ & 1.38 & $0.56-3.37$ & 0.478 \\
\hline Low $\mathrm{LDH}$ ratio $(<0.6)$ & 1 & reference & \\
\hline High LDH ratio $(>0.6)$ & 1.47 & $0.63-3.45$ & 0.372 \\
\hline No detected lung mass & 1 & reference & \\
\hline Lung mass detected by clinical imaging & 6.06 & $2.65-13.87$ & $<0.001^{*}$ \\
\hline No clinical extrathoracic metastasis & 1 & reference & \\
\hline Clinical extrathoracic metastasis & 1.08 & $0.54-2.19$ & 0.819 \\
\hline Lung cancer without extrathoracic metastasis & 1 & reference & \\
\hline Lung cancer with extrathoracic metastasis & 3.24 & $1.66-6.30$ & $0.001 *$ \\
\hline Single tap for pleural cytology & 1.23 & $0.51-2.95$ & 0.647 \\
\hline Double tap for pleural cytology & 1.88 & $0.68-5.15$ & 0.223 \\
\hline Multiple tap for pleural cytology & 1 & reference & \\
\hline Lung cancer & 13.26 & $4.31-40.78$ & $<0.001^{*}$ \\
\hline Breast cancer & 4.26 & $1.16-15.73$ & $0.029^{*}$ \\
\hline Other cancers & 1 & reference & \\
\hline
\end{tabular}

protein ratio, Pleural fluid protein / serum protein; LDH ratio, Pleural fluid LDH / serum LDH; *, statistical significant

was usually high in a malignant disease (Verma et al., 2016; Korczyński et al., 2018; Verma et al., 2016). This was a result of using glycolysis for energy in tumor cells instead of oxidative phosphorylation, a switch in the adenosine triphosphate (ATP) generating pathways, which was mediated by LDH (Pfeiffer et al., 2001; Goldman et al., 1964; Mansouri et al., 2017). Likewise, the infection caused by tuberculosis in the pleural fluid usually had a higher ADA secreted by mononuclear cells, lymphocytes, neutrophils, and red blood cells (Liang et al., 2008; Jiménez Castro et al., 2003).

However, the meta-analysis of using the cancer ratio for the diagnosis of MPE was based on the data from the PubMed and EMBASE databases The cancer ratio had a high diagnostic accuracy for predicting MPE. The pooled sensitivity and specificity of the cancer ratio were equal to 0.97 (95\% CI 0.92-0.99) and 0.89 (0.69-0.97) respectively; with AUC equal to 0.98 (95\% CI 0.97-0.99).

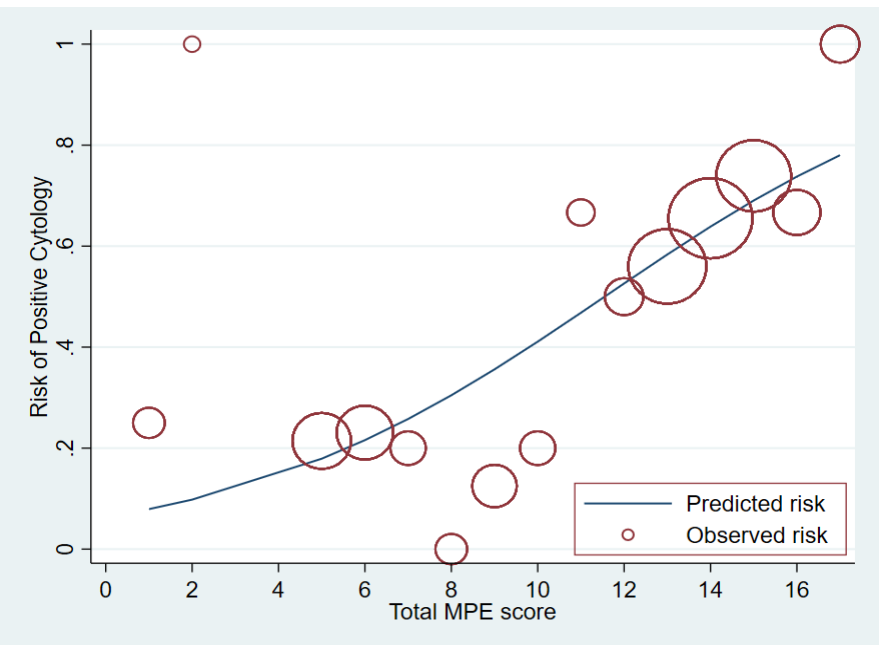

$\underline{\text { Logistic model for cyto, goodness-of-fit test }}$

(Table collapsed on quantiles of estimated probabilities) (There are only 8 distinct quantiles because of ties)

\begin{tabular}{|r|r|r|r|r|r|r|}
\hline Group & Prob & Obs_1 & Exp_1 & Obs_ $\theta$ & Exp_ $\theta$ & Total \\
\hline 1 & 0.1791 & 5 & 2.9 & 14 & 16.1 & 19 \\
2 & 0.2158 & 3 & 2.8 & 10 & 10.2 & 13 \\
3 & 0.3560 & 2 & 5.4 & 15 & 11.6 & 17 \\
4 & 0.5261 & 6 & 6.6 & 8 & 7.4 & 14 \\
5 & 0.5834 & 14 & 14.6 & 11 & 10.4 & 25 \\
\hline 7 & 0.6386 & 19 & 18.5 & 10 & 10.5 & 29 \\
9 & 0.6903 & 17 & 15.9 & 6 & 7.1 & 23 \\
10 & 0.7802 & 12 & 11.3 & 3 & 3.7 & 15 \\
\hline
\end{tabular}

$$
\begin{aligned}
\text { number of observations }= & 155 \\
\text { number of groups }= & 8 \\
\text { Hosmer-Lemeshow chi2 }(6)= & 5.45 \\
\text { Prob }>\text { chi2 }= & 0.4871
\end{aligned}
$$

Figure 2. Observed risk (circle) versus the score predicted risk (solid line) of the positive cytology pleural effusion (malignant pleural effusion). The size of the circle represents the frequency of MPE in each score (Left). Wellfitting model shows non-significance difference between the model and the observed data on the Hosmer-Lemeshow goodness of fit test (p-value 0.49) (Right). 
Table 3. Risk Score Derivation Using Multivariate Logistic Regression Coefficients.

\begin{tabular}{lccccc}
\hline Potential Predictors & Odds Ratio & 95\% Confidence Interval & p-value & Coefficients & Score \\
\hline Pleural fluid protein $<4.64 \mathrm{~g} / \mathrm{dL}$ & 1 & reference & - & - & 0 \\
Pleural fluid protein $>4.64 \mathrm{~g} / \mathrm{dL}$ & 1.27 & $0.63-2.56$ & 0.509 & 0.24 & 1 \\
Pleural fluid $\mathrm{LDH}<555 \mathrm{IU} / \mathrm{L}$ & 1 & reference & - & - & 0 \\
Pleural fluid $\mathrm{LDH}>555 \mathrm{IU} / \mathrm{L}$ & 1.27 & $0.59-2.72$ & 0.536 & 0.24 & 1 \\
Pleural fluid sugar $<60 \mathrm{mg} / \mathrm{dL}$ & 1 & reference & - & - & 0 \\
Pleural fluid sugar $>60 \mathrm{mg} / \mathrm{dL}$ & 3.31 & $1.15-9.52$ & 0.026 & 1.2 & 5 \\
No detected lung mass & 1 & reference & - & - & 0 \\
Lung mass detected by clinical imaging & 6.38 & $2.72-14.98$ & $<0.001$ & 1.85 & 8 \\
No double tap for pleural cytology & 1 & reference & - & - & 0 \\
Double tap for pleural cytology & 1.55 & $0.68-3.54$ & 0.298 & 0.44 & 2 \\
\hline
\end{tabular}

Table 4. The Sensitivity, Specificity, Positive Likelihood Ratio (LR+), and Negative Likelihood Ratio (LR-) of Each Cut-Off Point Value of the MPE Score

\begin{tabular}{lcccc}
\hline Cut-off Point & Sensitivity & Specificity & Positive Likelihood Ratio & Negative Likelihood Ratio \\
\hline 1 & $100.00 \%$ & $0.00 \%$ & 1.00 & 0.33 \\
2 & $98.72 \%$ & $3.90 \%$ & 1.03 & 0.66 \\
5 & $97.44 \%$ & $3.90 \%$ & 1.01 & 0.35 \\
6 & $93.59 \%$ & $18.18 \%$ & 1.14 & 0.33 \\
7 & $89.74 \%$ & $31.17 \%$ & 1.3 & 0.32 \\
8 & $88.46 \%$ & $36.36 \%$ & 1.39 & 0.28 \\
9 & $88.46 \%$ & $41.56 \%$ & 1.51 & 0.25 \\
10 & $87.18 \%$ & $50.65 \%$ & 1.77 & 0.25 \\
11 & $85.90 \%$ & $55.84 \%$ & 1.94 & 0.29 \\
12 & $83.33 \%$ & $57.14 \%$ & 1.94 & 0.34 \\
13 & $79.49 \%$ & $61.04 \%$ & 2.04 & 0.51 \\
14 & $61.54 \%$ & $75.32 \%$ & 2.49 & 0.71 \\
15 & $37.18 \%$ & $88.31 \%$ & 3.18 & 0.88 \\
16 & $15.38 \%$ & $96.10 \%$ & 3.95 & 0.92 \\
17 & $7.69 \%$ & $100.00 \%$ & & 1 \\
\hline 17 & $0.00 \%$ & $100.00 \%$ & & \\
\hline
\end{tabular}

Nevertheless, there were some limitations due to the bias of patient selection and potential partial verification (Han et al., 2019). Yet, it was frequently found that serum LDH may not be raised in the case of cancer. A high LDH may be related to poorer overall survival. Some minor studies (Chantharakit, 2018) also found that high LDH was related to cancer under liver metastasis; however, the data still contained a few limitations.

Porcel et al., (2004) used a panel of tumor markers, i.e., carcinoembryonic antigen (CEA), cancer antigen (CA) 125, carbohydrate antigen (CA) 15-3, and cytokeratin 19 fragments in pleural fluid for the differential diagnosis of benign and malignant effusions. The combination of the four tumor markers reached a sensitivity of $54 \%$, whereas the combined use of the cytology and the tumor marker panel increased the diagnostic yield of the former by $18 \%$ (95\% CI; 13-23\%). Yang et al., (2017) reported about a updated meta-analysis of patients with undiagnosed pleural effusion and showed that the combinations of positive pleural CEA + CA 15-3 and CEA + CA 19-9 were highly suspicious for pleural malignancy. Still, the sensitivity of these tests was poor.

Clive et al., (2014) studied prognostic indicators and found that the ones affecting the survival of MPE patients were pleural fluid LDH, the Eastern Cooperative Oncology Group (ECOG) performance status, and neutrophil-tolymphocyte ratio (NLR). It was also found that the tumor type could be developed by the LENT scoring system as a prognostic prediction model. The levels of pleural fluid LDH were key markers of inflammation or cellular injury.

Table 5. Internal Validation via 1,000 Resampling Bootstrap Method

\begin{tabular}{lccccc}
\hline Variable & Observe & Mean AUC & Standard Deviation & Min & Max \\
\hline Apparent C-statistics & 1,000 & 0.75 & 0.04 & 0.63 & 0.88 \\
Test C-statistics & 1,000 & 0.72 & 0.02 & 0.62 & 0.75 \\
Optimism C-statistics & 1,000 & 0.03 & 0.04 & -0.1 & 0.15 \\
\hline
\end{tabular}


LDH levels greater than three times the upper limit of normal (often $>1,000 \mathrm{U} / \mathrm{L}$ ) are often indicative of pleural infection. This can also be associated with rheumatoid pleurisy, tuberculous pleurisy or malignancy.

This study is different from previous studies as it focused only on PME. Pleural fluid cytology results may be positive malignant cells or negative malignant cells and it is not the intention of this study to distinguish MPE from benign disease. Therefore, all the pleural fluid was exudative pleural fluid according to Light's criteria. The clinical information fitting the malignant disease was used to find the predictive indicators affecting the diagnosis of MPE using pleural fluid cytology to develop a diagnostic prediction model (MPE score). This was the first diagnostic prediction model used to assist in the diagnosis of MPE in patients with cancer. The data from the pleural fluid biomarkers were used along with the clinical data of the patients rather than using only the data from the biomarkers.

However, the standard diagnosis of MPE features pleural fluid cytology supported by testing for confirmation by pleural biopsy in the case of negative pleural fluid cytology; still, with suspected MPE. This is an invasive procedure. Despite the effort to use a non-invasive technique, e.g., biomarker tests or molecular analysis from the pleural fluid for the diagnosis of MPE, the less invasive methods have not become popular yet. The validated clinical data found that there were still some limitations of use; thus, further studies are required.

Therefore, using the MPE score at the cut-off point of 15 , which has high specificity, may help in predicting MPE diagnosis to make decisions about planning for investigation while waiting for pleural fluid cytology results. This would enhance better efficiency of the diagnosis of MPE.

\section{Author Contribution Statement}

Chaichana Chantharakhit: Designed the study, reviewed the paper, collected data, analyzed data, and edited the final version. Nantapa Sujaritvanichpong: Collected data. All authors read and approved the final version.

\section{Acknowledgements}

The study protocol was approved by the Institutional Review Board of Buddhasothorn Hospital number BSHIRB $036 / 2563$. This research did not receive a specific grant from any funding agency in the public, commercial, or not-for-profit sectors. As such, there is no funding statement to declare.

\section{Funding Statement}

The authors confirm that there are no relevant financial or non-financial competing interests to report and no conflicts of interest to declare.

\section{Data Availability}

The data used to support the findings of this study have been deposited in the repository [https://drive.google.
com/drive/folders/1Enrqg5Zq_co3rY73-epiqymNe_ njLL7W?usp=sharing].

We confirm that there are no relevant financial or nonfinancial competing interests to report and no conflicts of interest to declare

\section{References}

Agrawal A, Tandon R, Singh L, Chawla A (2015). Clinicopathological profile and course of malignant pleural effusion in a tertiary care teaching hospital in western Uttar Pradesh with special reference to lung cancer. Lung India, 32, 678-9.

Ali MS, Light RW, Maldonado F (2019). Pleuroscopy or videoassisted thoracoscopic surgery for exudative pleural effusion: a comparative overview. J Thorac Dis, 11, 3207-16.

Antonangelo L, Sales RK, Corá AP, et al (2015). Pleural fluid tumour markers in malignant pleural effusion with inconclusive cytologic results. Curr Oncol, 22, e336-41.

Aydin Y, Turkyilmaz A, Intepe YS, Eroglu A (2009). Malignant pleural effusions: appropriate treatment approaches. Eurasian J Med, 41, 186-93.

Brock MV, Hooker CM, Yung R, et al (2005). Can we improve the cytologic examination of malignant pleural effusions using molecular analysis?. Ann Thoracic Surg, 80, 1241-7.

Chantharakit C (2018). The Correlation between Pretreatment Serum Lactate Dehydrogenase (LDH) Levels and various factors in Advanced Solid Tumor. Buddhasothorn Hospital J, 34, 1-6.

Clive AO, Kahan BC, Hooper CE, et al (2014). Predicting survival in malignant pleural effusion: development and validation of the LENT prognostic score. Thorax, 69 , 1098-104.

Desai NR, Lee HJ (2017). Diagnosis and management of malignant pleural effusions: state of the art in 2017. J Thorac Dis, 9, S1111-22.

Epelbaum O, Rahman NM (2019). Contemporary approach to the patient with malignant pleural effusion complicating lung cancer. Ann Transl Med, 7, 352.

Ferreiro L, Suárez-Antelo J, Valdés L (2017). Pleural procedures in the management of malignant effusions. Ann Thorac Med, 12, 3-10.

Garcia LW, Ducatman BS, Wang HH (1994). The value of multiple fluid specimens in the cytological diagnosis of malignancy. Mod Pathol, 7, 665-8.

Goldman R, Kaplan N, Hall T (1964). Lactic dehydrogenase in human neoplastic tissues. Cancer Res, 24, 389-99.

Han YQ, Zhang L, Yan L, et al (2019). Diagnostic accuracy of cancer ratio for malignant pleural effusion: a systematic review and meta-analysis. Ann Transl Med, 7, 554.

Herman JG, Graff JR, Myöhänen S, Nelkin BD, Baylin SB (1996). Methylation specific PCR: a novel PCR assay for methylation status of CpG islands. Proc Natl Acad Sci U S A, 93, 9821-6.

Hsu C (1987). Cytologic detection of malignancy in pleural effusion: a review of 5,255 samples from 3,811 patients. Diagn Cytopathol, 3, 8-12.

Jiménez Castro D, Díaz Nuevo G, Pérez-Rodríguez E, Light RW (2003). Diagnostic value of adenosine deaminase in nontuberculous lymphocytic pleural effusions. Eur Respir J, 21, 220-4.

Johnston WW (1985). The malignant pleural effusion. A review of cytopathologic diagnoses of 584 specimens from 472 consecutive patients. Cancer, 56, 905-9.

Korczyński P, Mierzejewski M, Krenke R, Safianowska A, Light RW (2018). Cancer ratio and other new parameters for 
differentiation between malignant and nonmalignant pleural effusions. Pol Arch Intern Med, 128, 354-61.

Liang QL, Shi HZ, Wang K, Qin SM, Qin XJ (2008). Diagnostic accuracy of adenosine deaminase in tuberculous pleurisy: a meta-analysis. Respir Med, 102, 744-54.

Loddenkemper R, Boutin C (1993). Thoracoscopy: present diagnostic and therapeutic indications. Eur Respir J, 6, $1544-55$.

Mansouri S, Shahriari A, Kalantar H, et al (2017).Role of malate dehydrogenase in facilitating lactate dehydrogenase to support the glycolysis pathway in tumors. Biomed Rep, 6, 463-7.

Mongardon N, Pinton-Gonnet C, Szekely B, et al (2011). Assessment of chronic pain after thoracotomy: a 1-year prevalence study. Clin J Pain, 27, 677-81.

Nakajima R, Abe K, Sakai S (2015). Diagnostic ability of FDG$\mathrm{PET} / \mathrm{CT}$ in the detection of malignant pleural effusion. Medicine (Baltimore), 94, e1010.

Pfeiffer T, Schuster S, Bonhoeffer S (2001). Cooperation and competition in the evolution of ATP-producing pathways. Science, 292, 504-7.

Porcel JM, Vives M, Esquerda A, et al (2004). Use of a panel of tumor markers (carcinoembryonic antigen, cancer antigen 125 , carbohydrate antigen $15-3$, and cytokeratin 19 fragments) in pleural fluid for the differential diagnosis of benign and malignant effusions. Chest, 126, 1757-63.

Psallidas I, Kalomenidis I, Porcel JM, Robinson B, Stathopoulos GT (2016). Malignant pleural effusion: From bench to bedside. Eur Respiratory Rev, 25, 189-98.

Starr RL, Sherman ME (1991). The value of multiple preparations in the diagnosis of malignant pleural effusions. A cost benefit analysis. Acta Cytol, 35, 533-7.

Toaff JS, Metser U, Gottfried M, Gur O, et al (2005). Differentiation between malignant and benign pleural effusion in patients with extra-pleural primary malignancies: assessment with positron emission tomography-computed tomography. Invest Radiol, 40, 204-9.

Valdes L, Alvarez D, Valle JM, Pose A, SanJose E (1996). The aetiology of pleural effusions in an area with highincidence of tuberculosis. Chest, 109, 158-62.

Verma A, Abisheganaden J, Light RW (2016). Identifying malignant pleural effusion by a cancer ratio (Serum LDH: Pleural Fluid ADA Ratio). Lung, 194, 147-53.

Verma A, Dagaonkar RS, Marshall D, Abisheganaden J, Light RW (2016). Differentiating malignant from tubercular pleural effusion by Cancer Ratio Plus (Cancer Ratio: pleural lymphocyte count). Can Respir J, 2016, 7348239.

Wong FM, Grace WJ, Rottino A (1963). Pleural effusions, ascites, pericardial effusions and edema in Hodgkin's disease. Am J Med Sci, 246, 678-82.

Yang Y, Liu YL, Shi HZ (2017). Diagnostic accuracy of combinations of tumor markers for malignant pleural effusion: An Updated Meta-Analysis. Respiration, 94, 62-9.

Zhang X, Zhao Y, Wang M, Yap WS, Chang AY (2007).Detection and comparison of epidermal growth factor receptor mutations in cells and fluid of malignant pleural effusion in non-small cell lung cancer. Lung Cancer, 60, 175-82.

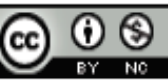

This work is licensed under a Creative Commons AttributionNon Commercial 4.0 International License. 